\title{
Von Studenten und Dozenten
}

Erhard Taverna

erhard.taverna@saez.ch
Demonstrationen und Anschläge von Anarchisten begleiten die aktuelle Universitätsreform in Italien. Ein Artikel im Corriere della Sera zitiert den ehemaligen Staatspräsidenten Luigi Einaudi, wonach die Diplome und Lizentiate weniger wert seien als das Papier, auf dem sie geschrieben wurden.

Im nachweihnachtlichen Bologna sind die Studenten in den Winterferien. Eine frostige Sonne liegt auf den ockerfarbenen Hausfassaden und den endlosen Arkaden der Altstadt. Im alten Glanz erstrahlen auch die ehemaligen Zentren der Gelehrsamkeit mit ihren wappengeschmückten Treppenhäusern und Hörsälen. Bekannte Namen der Zoologie, Physik, Astronomie und Medizin erinnern an die Beiträge mehrerer Lehrergenerationen zum wissenschaftlichen Fortschritt Europas.

Mit dem «Istituto delle Scienze» im Palazzo

Poggi wurde 1711 das welterste Lehrzentrum eingerichtet, das mit festbesoldeten Dozenten, einer Sternwarte und gutausgerüsteten Labors in vorbildlicher Weise Lehre und Forschung vereinigte. Zu den damaligen Sponsoren gehörten neben reichen Adligen auch der Papst und der Stadtsenat, der das Gebäude kaufte. Allein die vielen prunkvollen Säle mit ihren Wand- und Deckengemälden lohnen den Besuch. Von unschätzbarem Wert sind die aufwendig restaurierten Privatsammlungen früherer Gelehrter. Sie illustrieren den langen Weg vom Raritätenkabinett zu den systematischen Beobachtungen eines Ulisse Aldrovandi (1522-1605) als unverzichtbare Grundlage des heutigen Weltverständnisses. $\mathrm{Zu}$ jedem ausgestellten Objekt gehören kunstvoll gefertigte Holzschnitte, die teilweise nach Exkursionen mit Studenten entstanden. Das drucktechnisch verbreitete Wissen begründete den Ruhm des Autors als Wegbereiter der modernen Zoologie. Für medizinisch Interessierte haben die «Cere anatomiche» eine zentrale Bedeutung. Mit den lebensgrossen Wachs- und Tonfiguren von Reproduktionsorganen und Feten modernisierte der Chirurg Giovanni Antonio Galli (1708-1782) die Geburtshilfe für Ärzte und Hebammen. Die Vitrinen zeigen Modelle aller möglichen Geburtspathologien wie regelwidrige Kopfstände, Deflexions- und Strecklagen, Nabelschnur- und Armvorfälle, Mehrlinge und
Placentaanomalien. An der Geburtsmaschine, einem hölzernen Becken mit gläsernem Uterus, übten die Studenten mit verbundenen Augen die korrekte Palpation und Extraktion unter der persönlichen Aufsicht des Erfinders. Ein geburtshilflicher Stresstest, der auch meinen Jahrgang am Ledermodell der Universitätsklinik in Zürich das Gruseln lehrte.

Berühmter wurde sein Nachfolger Luigi Galvani (1737-1798), dessen Experimente mit Froschschenkeln die elektrochemische Physiologie einleiteten. Notabene mit Hilfe der ersten gewählten Professorin in Europa, der Physikerin Laura Bassi (17111778). Ein Experiment, das mit der Dekapitation von Fröschen mittels einer Schere zu den weniger beliebten Fortsetzungen meines propädeutischen Lernens zählte. Im Kellergeschoss des «Museo Civico Medievale», nahe der Piazza Maggiore, lagern die Grabplatten und Monumente der Dozenten und ihrer Studenten. Reliefs und Statuen zeigen der Nachwelt die Lehrer und Schüler, eingezwängt in Bänken, Kathetern und Pulten, fleissig am Schreiben, das Kinn aufgestützt, gelangweilt oder interessiert. Eine in Stein gemeisselte Ermahnung, dass akademische Ehren ohne ausdauerndes Sitzen nicht zu haben sind.

Die Problemliste, so der Kommentar im Corriere della Sera, sei lang. Zu viele Universitäten im Lande: «ne sono 95, perché, sotto la spinta partitico-sindacal-corporativa, si è costruita una università accanto ad ogni campanile», fehlende Bewertung, desinteressierte, den Unterricht schwänzende Studenten und zu viele schlechte Dozenten. Das Wahlsystem sei eine Farce, da die Wahlkommissionen stets nur die hauseigenen, genehmen Kandidaten wählten, und seien diese noch so schlecht: «Così sono saliti sulla cattedra centinaia e centinaia di mediocri o addirittura di asini.» [1]

Gut zu wissen, dass bisher den schlechten auch immer wieder gute Zeiten folgten.

1 Giuseppe Bedeschi. La svolta è liberare l'università dalla logica del «pezzo di carta». Corriere della Sera vom 3.12.2010. 